\title{
Leiomyosarcoma in leiomyomatosis of the small intestine
}

\author{
Magdi El-Omar, Jason Davies, Sandeep Gupta, Harvey Ross and \\ Richard Thompson
}

Gastrointestinal Laboratory, The Rayne Institute, St Thomas' Hospital, London SE1 7EH, UK

\begin{abstract}
Summary: Multiple leiomyomata of the small intestine are rare. We report one such case where a leiomyosarcoma had arisen from a leiomyoma in the small intestine 8 years after presentation. The possible origin of the leiomyomata is discussed and it is concluded that small intestinal leiomyomatosis should be regarded as a premalignant condition.
\end{abstract}

\section{Introduction}

Smooth muscle tumours of the small intestine account for less than $1 \%$ of all gastrointestinal tumours.' Half are benign and usually solitary. Rarely they may be multiple, growing either discretely or in a diffuse fashion. This is termed small intestinal leiomyomatosis and has been regarded as a benign condition. ${ }^{2}$ However, we present a case of discrete small intestinal leiomyomatosis in which malignant transformation occurred 8 years after presentation.

\section{Case report}

A 40 year old woman presented in 1981 with iron deficiency anaemia and an acute abdomen. At laparotomy the appendix was inflamed, but in addition there were multiple intramural and submucosal polyps throughout the entire small intestine. One large polyp at the jejuno-ileal junction was resected, together with a cuff of bowel that contained a subserosal nodule. Histological examination of the polyp showed it to consist partly of spindle-shaped pale eosinophilic or clear cells, and partly of oval or round cells, with clear cytoplasm. In some areas there was palisading of the cell nuclei with a tendency of the cells to stream in parallel bundles. The tumour appeared to arise from the muscularis propria and occupied most of the thickness of the bowel wall, with central ulceration on the luminal surface. Mitoses were rare with less than one per ten high power field (HPF). The appearances were those of a benign leiomyoma; the subserosal nodule was similar. Small bowel enemata were performed every 6 months, with no increase in size of the remaining tumours, but after 3 years the patient was lost to follow-up.

She remained asymptomatic until she represented in 1989 with abdominal pain, iron deficiency anaemia and fever. Examination revealed symmetrical pigmentary changes on the perineum and groins (Figure 1). The pigmentation, which had been present since her teens, was speckled at the margins and confluent adjacent to the vulva, extending in an hour-glass pattern around the

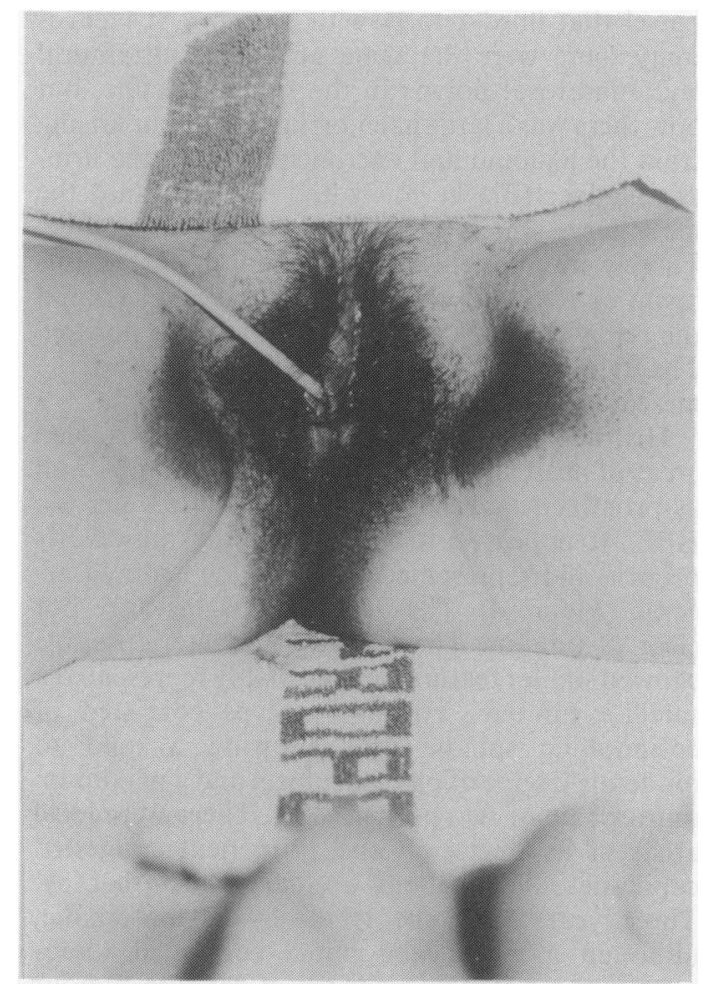

Figure 1 Abnormal pigmentation in the perineum. 


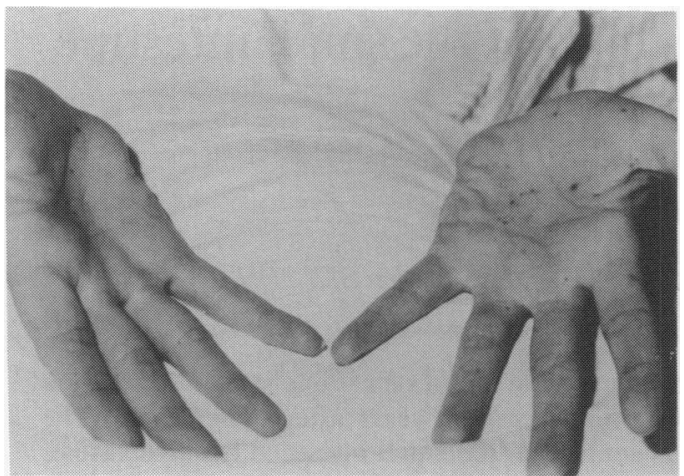

Figure 2 Pigmented lesions on the palms.

introitus and anus. The labia minora, introitus and rectal mucosa were normal. She also had a number of pigmented lesions on the palms (Figure 2), but none around or inside the mouth. Serum levels of adrenocorticotrophic hormone, $\beta$-melanocyte stimulating hormone, cortisol, aldosterone, renin, follicle stimulating hormone and luteinizing hormone and thyroid function tests, chest X-ray and chromosomal karyotyping were all normal. A small bowel enema revealed a $12 \mathrm{~cm}$ segment of bowel that filled poorly with barium. At laparotomy there were the same numerous intramural and subserosal polyps in the small intestine, but now there was a large haemorrhagic tumour arising from the jejunum and encroaching upon the urinary bladder (Figure 3 ). It had extended into the mesentery, which contained a separate nodule. There were no metastases in lymph nodes, peritoneum or liver and the uterus, ovaries, spleen and the remaining gastrointestinal tract were normal. The tumour, two further small polyps and the mesenteric nodule were resected.

Histological examination of the tumour revealed areas of necrosis and myxoid degeneration, as well as prominent palisading with ten mitoses per ten HPF. It appeared to arise from the muscularis propria and represented a high grade leiomyosarcoma (Figure 4). The resected margins were not clear of tumour. The separate mesenteric nodule showed similar features and probably represented a satellite tumour. The two polyps consisted of eosinophilic spindle cells showing a mild to moderate degree of pleomorphism and a maximum mitotic rate of two per ten HPF. There were local areas of hyalinization and prominent congested capillaries. The patient declined chemotherapy. Three years later she is totally asymptomatic, although a recent abdominal computed tomographic scan reveals evidence of satellite tumour throughout her abdominal cavity.

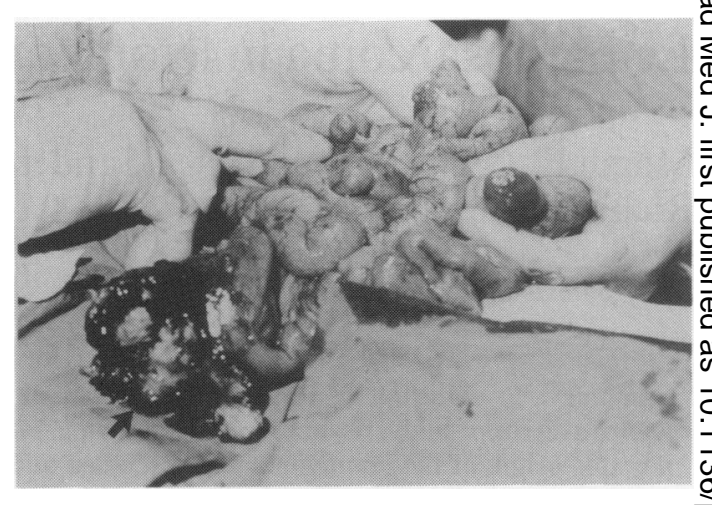

Figure 3 Small intestine with multiple subserosal polyps and a large haemorrhagic tumour (arrow).

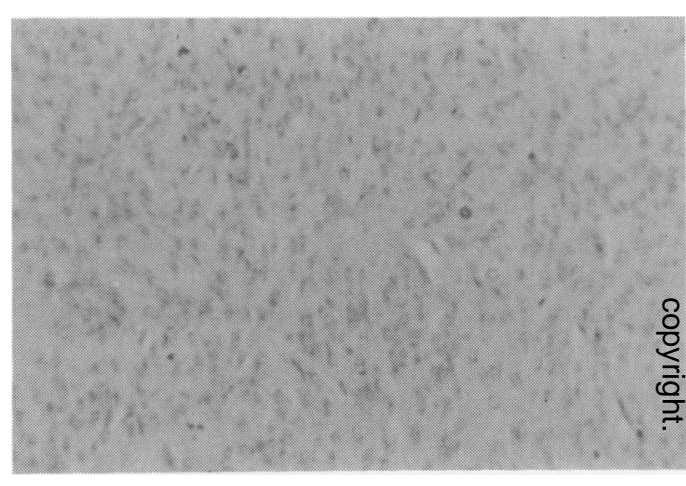

Figure 41989 tumour composed of pleomorphic cells, including several mitotic figures, representing a leiomyosarcoma $(\mathrm{H} \& \mathrm{E}, \times 400)$.

\section{Discussion}

Small bowel tumours account for only $3-4 \%$ of all gastrointestinal tumours; $20 \%$ are smooth muscle tumours, of which half are histologically benign., They are usually solitary and may present with iron deficiency anaemia, a palpable mass, abdominal pain, fever or intestinal obstruction. ${ }^{3}$ Multiple benign smooth muscle tumours in the small intestine are rare. There have been only four documented cases of small intestinal leiomyomatosis. ${ }^{2,4-6}$ In three the leiomyomata were discrete and occupied a short segment of small intestine, but in the fourth ${ }^{2}$ the leiomyomata formed confluent intramural nodules arising in the small intestinal and colonic walls resembling diffuse leiomyomatosis of the oesophagus and colon. ${ }^{7,8}$ Malignant transformation was not reported in any of these cases nor in any other reports of leiomyomatosis of the digestive tract, leading Vallaeys et al. to suggest that this condition follows a benign course. ${ }^{2}$ 
In the present case, the leiomyomata occupied the whole length of the small intestine in a nonconfiuent fashion. In addition, malignant transformation was demonstrated 8 years after presentation. This may represent a case of primary small intestinal leiomyomatosis with subsequent development of a leiomyosarcoma in a previously benign leiomyoma. Alternatively, the original tumours may have been metastases from a small bowel primary, although the low mitotic rate $(0.5$ per ten HPF) and the prolonged survival of the patient with no noted increase in tumour size are against this. In addition, metastases should involve other sites. However, predicting the behaviour of gastrointestinal smooth muscle tumours is difficult, and different from smooth muscle tumours at other sites, such as the uterus and soft tissues.

Nevertheless, the single most important factor is the number of mitotic figures. Evans found this to be the only prognostically significant factor and divided leiomyosarcomas into high grade (ten or more mitoses per ten HPF) and low grade (less than ten per ten HPF - actual rates 1-5 per ten HPF) using the mitotic count alone. ${ }^{9}$ Ranchod and Kempson reported aggressive behaviour in all those tumours with five or more mitoses per ten HPF, but found that $40 \%$ of all leiomyosarcomas had actual mitotic rates less than this. ${ }^{10}$ In the absence of a high mitotic rate, tumour cell necrosis was associated with aggressive behaviour. With neither of these factors, prediction became difficult. Taken together, a worse prognosis was seen in large tumours $(>5 \mathrm{~cm})$ with increased cellularity and cellular atypia. Haemorrhage and mucosal ulceration are also more frequent in malignant tumours, with myxoid change, hyalinization and calcification in benign tumours. Metastatic uterine leiomyosarcoma and benign metastasizing leiomyomatosis $^{11}$ were excluded by a normal uterus and the absence of leiomyomata in the lungs, skin or lymph nodes. Leiomyomatosis peritonealis disseminata, a rare non-neoplastic proliferation of smooth muscle in the abdominal cavity, usually in women of child-bearing age, was excluded by the absence of decidual cells mixed with the muscle. In addition, it is unknown for this condition solely to affect the small intestine without widespread involvement of the peritoneum. ${ }^{12}$

\section{References}

1. Braasch, J.W. \& Denbo, H.E. Tumors of the small intestine Surg Clin North Am 1964, 44: 791.

2. Vallaeys, J.H., Cuvelier, C.A., Bekaert, L. \& Roels, H. Combined leiomyomatosis of the small intestine and colon. Arch Pathol Lab Med 1992, 116: 281-283.

3. He, L.J., Wang, B.S. \& Chen, C.C. Smooth muscle tumours of the digestive tract: report of 160 cases. Br J Surg 1988, 75: 184-186.
The pigmentary change seen in this patient was also present in the patient's eldest daughter, aged 24 , in whom it also appeared in late teens. None of the remaining three daughters had similar pigmentation. The association between leiomyomatosis and abnormal pigmentation has not been described before and they may not be related. However, similar associations exist in other conditions such as the Peutz-Jehgers and Cronkhite-Canada syndromes, which have a different distribution of abnormal pigmentation; they are not associated with smooth muscle tumours. ${ }^{13.14}$ There are, in addition, two case reports of small intestinal leiomyosarcoma associated with abnormal mucocutaenous pigmentation. ${ }^{15,16}$ The presence of similar pigmentary change in the daughter raises the question of whether she has, or will develop at a later stage, similar gastrointestinal pathology to that found in her mother, perhaps as part of a family cancer syndrome. The latter, however, is unlikely in view of a negative family history for any cancers and normal chromosomal karyotyping in the mother. Despite careful counselling, the patient's daughter did not wish to have any investigations.

When leiomyomata of the small intestine are solitary or confined to a single area of small bowel, it is perhaps unjustified to follow the patients up by regular barium studies or computed tomographic scans. However, when the tumours are disseminated throughout the whole small intestine, the cumulative risk of malignant transformation becomes significant and regular follow-up is indicated.

We conclude that leiomyomatosis of the small intestine is a potentially premalignant condition which warrants long-term follow-up. Tumour should not be labelled as benign or malignant as many histologically benign tumours behave aggressively. Abnormal mucocutaneous pigmentation may in addition be a marker of digestive tract leiomyomatosis.

\section{Acknowledgement}

We acknowledge the excellent secretarial assistance of Sue Rothwell.

4. Nagar, R.C., Mehta, J.M. \& Bhargava, K.N. Multiple leiomyomas of small intestine. $J$ Ind Med Assoc 1982, 78: 49-50.

5. Bennett, R.C. Intestinal tumor simulating leiomyomas of the uterus. Am J Obs Gyn 1963, 87: 131-132. 
6. Cox, J.G.C., Royston, C.M.S. \& Sutton, D.R. Multiple smooth muscle tumors in neurofibromatosis presenting with chronic gastrointestinal bleeding. Postgrad Med J 1988, 64: 149-151.

7. Heald, J., Moussalli, H. \& Hasleton, P.S. Diffuse leiomyomatosis of the oesophagus. Histopathology 1986, 10: 755-759.

8. Freni, S.C. \& Keeman, J.N. Leiomyomatosis of the colon Cancer 1977, 37: 263-266.

9. Evans, H.L. Smooth muscle tumors of the gastrointestinal tract. Cancer 1985, 56: 2242-2250.

10. Ranchod, M.\& Kempson, R.L. Smooth muscle tumors of the gastrointestinal tract and retroperitoneum. Cancer 1977, 39: 255-262.

11. Steiner, P. Metastasising fibroleiomyoma of the uterus. Report of a case and review of the literature. Am J Pathol 1939, 15: 89-107.
12. Williams, L.J. \& Paylick, F.J. Leiomyomatosis peritonealis disseminata. Cancer 1980, 45: 1726-1733.

13. Dormandy, T.L. Gastrointestinal polyposis with mucocutaneous pigmentation (Peutz-Jeghers syndrome). $N$ Engl J Med 1957, 256: 1093-1102.

14. Cronkhite, L.W. \& Canada, W.J. Generalised gastrointestinal polyposis: unusual symptoms of polyposis, pigmentation, alopecia and onychotrophia. $N$ Engl J Med 1955, 252: 1011-1015.

15. Suda, M., Shii, H., Kashiwazaki, K. \& Tsuchiya, M. Hyperpigmentation of skin and nails in a patient with intestinal leiomyosarcoma. Dig Dis Sci 1985, 30: 1108-1111.

16. Babin, R.W., Ceilly, R.I: \& DeSanto, L.W. Oral hyperpigmentation and occult malignancy - report of a case. $J$ Otolaryngol 1978, 7: 389-394. 